\title{
Metabolism of acetaminophen (paracetamol) in plants - two independent pathways result in the formation of a glutathione and a glucose conjugate
}

\author{
Christian Huber • Bernadett Bartha • \\ Rudolf Harpaintner • Peter Schröder
}

Received: 22 August 2008 / Accepted: 30 November 2008 / Published online: 15 January 2009

(C) The Author(s) 2009. This article is published with open access at Springerlink.com

\begin{abstract}
Background, aim, and scope Pharmaceuticals and their metabolites are detected in the aquatic environment and our drinking water supplies. The need for high quality drinking water is one of the most challenging problems of our times, but still only little knowledge exists on the impact of these compounds on ecosystems, animals, and man. Biological waste water treatment in constructed wetlands is an effective and low-cost alternative, especially for the treatment of non-industrial, municipal waste water. In this situation, plants get in contact with pharmaceutical compounds and have to tackle their detoxification. The mechanisms for the detoxification of xenobiotics in plants are closely related to the mammalian system. An activation reaction (phase I) is followed by a conjugation (phase II) with hydrophilic molecules like glutathione or glucose. Phase III reactions can be summarized as storage, degradation, and transport of the xenobiotic conjugate. Until now, there is no information available on the fate of pharmaceuticals in plants. In this study, we want to investigate the fate and metabolism of $\mathrm{N}$-acetyl-4-aminophenol (paracetamol) in plant tissues using the cell culture of Armoracia rusticana $\mathrm{L}$. as a model system.

Materials and methods A hairy root culture of A. rusticana was treated with acetaminophen in a liquid culture. The formation and identification of metabolites over time were analyzed using HPLC-DAD and $\mathrm{LC}-\mathrm{MS}^{\mathrm{n}}$ techniques.

Results With LC-MS technique, we were able to detect paracetamol and identify three of its metabolites in root
\end{abstract}

C. Huber $\cdot$ B. Bartha $\cdot$ R. Harpaintner $\cdot$ P. Schröder $(\bowtie)$

Department of Microbe-Plant Interactions,

German Research Center for Health and Environment GmbH,

Ingolstädter Landstraße 1,

85764 Neuherberg, Germany

e-mail: peter.schroeder@helmholtz-muenchen.de cells of $A$. rusticana. Six hours after incubation with $1 \mathrm{mM}$ of acetaminophen, the distribution of acetaminophen and related metabolites in the cells resulted in $18 \%$ paracetamol, $64 \%$ paracetamol-glucoside, $17 \%$ paracetamol glutathione, and $1 \%$ of the corresponding cysteine conjugate.

Discussion The formation of two independently formed metabolites in plant root cells again revealed strong similarities between plant and mammalian detoxification systems. The detoxification mechanism of glucuronization in mammals is mirrored by glucosidation of xenobiotics in plants. Furthermore, in both systems, a glutathione conjugate is formed. Due to the existence of $\mathrm{P} 450$ enzymes in plants, the formation of the highly reactive NAPQI intermediate is possible.

Conclusions In this study, we introduce the hairy root cell culture of $A$. rusticana $L$. as a suitable model system to study the fate of acetaminophen in plant tissues. Our first results point to the direction of plants being able to take up and detoxify the model substrate paracetamol. These first findings underline the great potential of using plants for waste water treatments in constructed wetlands.

Recommendations and perspectives This very first study on the detoxification of a widely used antipyretic agent in plant tissues again shows the flexibility of plant detoxification systems and their potential in waste water treatment facilities. This study covers only the very first steps of acetaminophen detoxification in plants; still, there is no data on long-term exposure as well as the possible impact of pharmaceuticals on the plant health and stress defense. Long-term experiments need to be performed to follow the fate of acetaminophen in root and leaf cells in a whole plant system, and to evaluate possible usage of plants for the remediation of acetaminophen from waste water.

Keywords Acetaminophen · Glucoside · LC-MS . Paracetamol $\cdot$ Pharmaceuticals $\cdot$ Phytoremediation 


\section{Background, aim, and scope}

Pharmaceuticals in the aquatic environment became an issue of increasing importance over the last years. Increasing proportions of all prescribed pharmaceuticals are excreted into sewage and treated in waste water treatment plants. Current literature proves that significant amounts of the active compounds or the first metabolites can be detected throughout the world at concentrations in the $\mathrm{ng} / \mathrm{l}$ to $\mu \mathrm{g} / \mathrm{l}$ range, even in the best available treatment systems (Kolpin et al. 2002; Schröder et al. 2007). Being designed as biologically active compounds, even the lowest concentrations of medicaments might have an impact on human health or on the aquatic food web, considering the potential of chronic exposure (Salomon 2005). One option for the removal of residual and recalcitrant compounds from sewage water is the implementation of filter dams and lagoons with wetland plant species, or aquatic macrophytes (Schröder et al. 2008). Although known and proposed for more than a decade, phytoremediation of pharmaceuticals is scarcely applied and, until now, only limited data on the ecotoxicological effects of pharmaceutical substances and especially their effects on plants exist. Acetaminophen (paracetamol) is a widely spread analgesic, anti-inflammatory, and antipyretic agent in human medicine and one of the possible problem compounds in water bodies (SchulteOehlmann et al. 2007; Crane et al. 2006). Based on ecotoxicological studies by Henschel et al. (1997) with algae, Daphnia, fish embryos, and bacteria, paracetamol is classified as harmful to aquatic organisms according to the existing German legislation (Gefahrenstoffverordnung). Considering its chemical structure, it is a benzene ring substituted by a hydroxyl group and an amide group in the $(1,4)$ para pattern. With its two activating groups, the benzene ring is highly reactive towards electrophilic aromatic substitution. In mammals, paracetamol is detoxified in the liver where, at low doses, sulfation and glucoronidation result in the formation of the inactive, non-toxic sulfate and glucuronid conjugates (Scheme 1). Toxication occurs when high concentrations of the drug are ingested. Under these conditions, $N$-acetyl- $p$-benzoquinoneimine (NAPQI), a reactive metabolite that interacts with proteins and nucleic acids, can be formed. Still, this highly reactive metabolite is conjugated to the nucleophile, reduced glutathione (Bertolini et al. 2006). This reaction is catalyzed by glutathione $S$-transferases (GSTs, E.C. 2.5.1.18) which accelerate glutathione conjugation and thereby usually serve to protect the cell. Next to the conjugation of artificial substrates, GSTs are involved in the conjugation of secondary metabolites and in the detoxification of metabolites that are formed under the conditions of oxidative stress (Edwards and Dixon 2004). Glucuronyl conjugates represent one of the most prominent groups of phase II metabolites in mammalians. A wide range of chemicals can serve as acceptor molecules for glucuronic acid if they carry an appropriate functional group. Uridine 5 '-diphophoglucuronic acid (UDP-glucuronic acid) acts as a cosubstrate for the reaction and is generated in the cytosol. Comparably, in plants, the glycosylation of small molecules by glycosyltransferases (E.C. 2.4) is a key mechanism in the metabolic homoeostasis of plant cells. Potential acceptor molecules can be plant hormones (Hou et al. 2004), endogenous synthesized secondary metabolites (Richman et al. 2005) as well as xenobiotics (Messner et al. 2003). With their multiple functions and the variety of possible substrates, glycosyltransferases play a major role in buffering the impacts of either biotic or abiotic challenges on the plant (Bowles et al. 2005). The present study aimed at comparing the detoxification mechanisms for acetaminophen in mammals and plants by studying its fate and metabolism in the hairy root cell culture of Armoracia rusticana L. as a model system, and to give recommendations for the removal of the pharmaceutical from waste water by phytotechniques. A. rusticana hairy roots are an easy-to-handle culture allowing exact dosage and ensuring equal distribution throughout the tissue. Using this culture seemed favorable because transport, storage, and distribution phenomena in a whole plant would be far more complex and require special discussion. Furthermore, leaf metabolism seems to be very different from root metabolism.

\section{Materials and methods}

\subsection{Plant material}

A hairy root culture of horseradish (A. rusticana L.) that was transformed by Agrobacterium rhizogenes strain A4 as described (Nepovim et al. 2004) was grown in Erlenmeyer flasks in 100-ml full-strength Murashige and Skoog medium containing thiamine and inositol for 10 days. Acetaminophen was dissolved in ethanol and set to a final concentration of $1 \mathrm{mM}$ in the growth medium. Incubation with paracetamol lasted for $3 \mathrm{~h}$. Root cells were washed twice with deionized and tap water and then transferred into fresh growth medium. Samples were taken at 0, 2, 4, and $6 \mathrm{~h}$ after incubation. Cells were dried on lint tissue paper, immediately frozen in liquid nitrogen, and stored at $-80^{\circ} \mathrm{C}$.

2.2 Extraction of acetaminophen and metabolites and sample preparation

For the extraction of acetaminophen and its metabolites, $0.5 \mathrm{~g}$ root cells were homogenized under liquid nitrogen 


\section{Acetaminophen-Glucuronid}

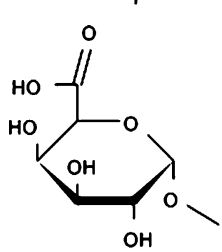

mammalians<smiles>CNc1ccc(F)cc1</smiles>

ins<smiles>CCC(C)=O</smiles>

Glucuronisation

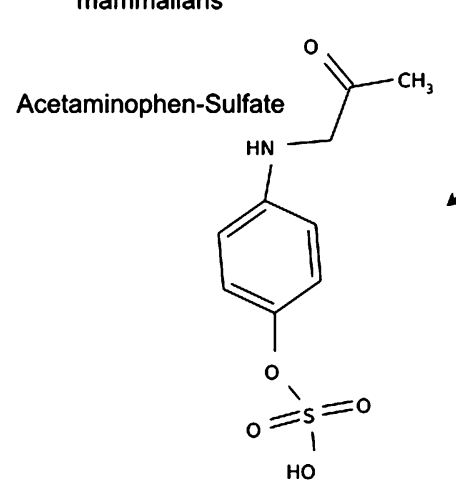

mammalians

Acetaminophen-Glucoside

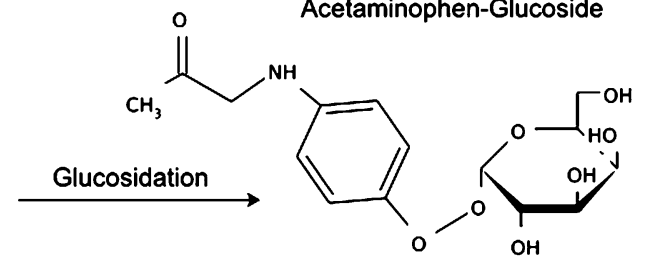

plants, fungi gi

HO

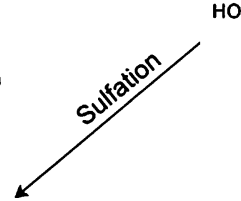

Acetaminophen<smiles>CC(=O)CNc1ccc(C)cc1</smiles>
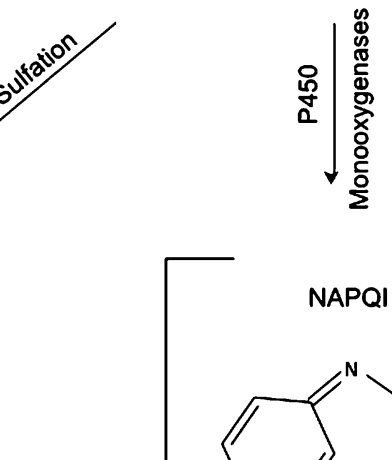

(NAPQI

$\longrightarrow$ Reaction with proteins ans nucleic acids

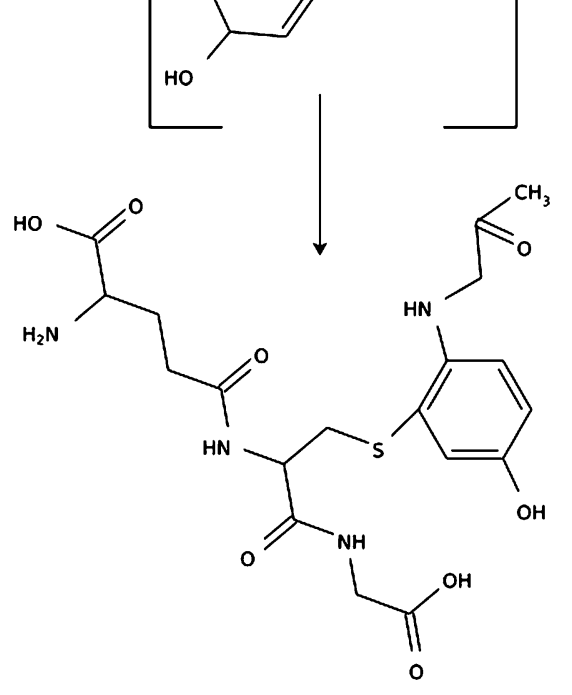

Acetaminophen-Glutathione: mammalians, plants

Scheme 1 Proposed metabolic pathways of acetaminophen

in $1 \mathrm{ml} 0.1 \mathrm{M} \mathrm{HCl}$. Acetanilide was used as an internal standard and added to the sample before solid phase extraction (SPE) at a concentration of $100 \mu \mathrm{M}$. After centrifugation with $18,000 \times g$ at $4^{\circ} \mathrm{C}$ for $30 \mathrm{~min}$, the supernatant was loaded on SPE columns (Phenomenex strata-X, $300 \mathrm{mg}$ ) that were preconditioned with $1 \mathrm{ml}$ of methanol and $1 \mathrm{ml}$ of water. After loading $500 \mu \mathrm{l}$ of root extract, columns were washed with $1 \mathrm{ml}$ of $5 \%$ methanol to remove impurities. Analytes were eluted with $500 \mu \mathrm{l}$ acetonitrile/methanol $(50 / 50, v / v)$ under gravity with a flow rate of less than $1 \mathrm{ml} / \mathrm{min}$. For HPLC and LC-MS analysis, this fraction was further diluted with water (MilliQ) by a factor of $10(\mathrm{v} / \mathrm{v})$. For the incubation of metabolites with $\beta$-D-glucosidase (E.C. 3.2.1.21), an additional extraction was carried out using $50 \mathrm{mM} \mathrm{Na}$ - phosphate buffer ( $\mathrm{pH}$ 5.0). The efficiency of the extraction was confirmed by the quantification of acetaminophen in roots extracts using either one or the other extraction buffer.

\subsection{HPLC analysis}

The content of acetaminophen in the samples was determined via HPLC (Varian ProStar 215) under reversed phase conditions, applying a linear gradient of eluents (buffer A $\mathrm{H}_{2} \mathrm{O}, 0.1 \%$ TFA; buffer B acetonitrile, $0.1 \%$ TFA) with a C18 Hypersil-ODS $(5 \mu \mathrm{m}, 250 \times 4.6 \mathrm{~mm})$ column and a flow rate of $1 \mathrm{ml} / \mathrm{min}$. Samples were filtered before injection. Injection volume was $40 \mu \mathrm{l}$. Detection of acetaminophen was carried out at $250 \mathrm{~nm}$ using a diode 
array detector (Varian ProStar 335). Identification of acetaminophen was done by comparison of the spectra and the retention time of an authentic paracetamol standard. A calibration curve for acetaminophen was used for the quantification of the content of acetaminophen in root cells and in the growth medium.

\subsection{LC-MS analysis}

LC-MS analysis was carried out on a HPLC system (Varian ProStar 210) that was coupled to an ion trap mass spectrometer (Varian 500-MS). The HPLC eluent was introduced to the mass spectrometer using a pneumatically assisted electrospray source. The mass spectrometer was operated in the positive ion mode. The interface was adjusted to the following conditions: capillary voltage, $40 \mathrm{~V}$; needle voltage, 4,000 V; sprayshield voltage, $600 \mathrm{~V}$; nebulizer gas pressure, $50 \mathrm{psi}$; drying gas pressure, $30 \mathrm{psi}$; drying gas temperature, $300^{\circ} \mathrm{C}$. A $5-\mu 1$ aliquot of each sample was directly injected into the HPLC system. MS/ MS spectra were obtained by collision-induced dissociation using helium as the collision gas. The mass transitions for the internal standard, acetaminophen and its metabolites were $136 \rightarrow 94$ (acetanilide, internal standard), $152 \rightarrow 110$ (acetaminophen), $457 \rightarrow 328$ (glutathione conjugate), $328 \rightarrow 182$ (cysteine conjugate), and $314 \rightarrow$ $152 \rightarrow 110$ (glucoside conjugate). The peak areas from each of these transitions were obtained, and ratios of the analytes to the internal standard were calculated for every sample.

The HPLC conditions were as follows: the column was a Phenomenex HYDRO-RP column (C18, polar endcapped; particle size $5 \mu \mathrm{m} ; 4.6 \times 200 \mathrm{~mm}$ ID). Paracetamol and its metabolites were separated using a linear gradient of eluents: buffer $\mathrm{A}\left(\mathrm{H}_{2} \mathrm{O}, 0.1 \%\right.$ formic acid) and buffer B (acetonitrile, $0.1 \%$ formic acid). Elution gradient: 0-2 min 97\% buffer A (isocratic); 2-10 min 95\% buffer B (linearly increasing); 10-12.5 min 95\% buffer B (isocratic); $12-12.5$ min 97\% buffer A (linear decreasing); $12.5-17 \mathrm{~min} 97 \% \mathrm{~A}$ (isocratic). The flow rate was $0.3 \mathrm{ml} / \mathrm{min}$.

All solvents used for LC-MS were of the highest grade available.

\subsection{Enzymatic hydrolysis}

Aliquots of $400 \mu \mathrm{l}$ of the extract prepared with $50 \mathrm{mM}$ Na-phosphate buffer were incubated with $100 \mathrm{U}$ of $\beta$ D-glucosidase at $36^{\circ} \mathrm{C}$ for $1 \mathrm{~h}$. The incubation was stopped by loading the samples on SPE columns. Control experiments were carried out concurrently without $\beta$-D-glucosidase. Samples were analyzed with LC-MS/MS.

\section{Results}

\subsection{Uptake of acetaminophen}

To evaluate the uptake of acetaminophen during the $3 \mathrm{~h}$ of incubation, samples of root cells and growth medium were taken right after $0,1,2$, and $3 \mathrm{~h}$ after the addition of acetaminophen. The concentration of non-conjugated paracetamol in the cells and in the incubation medium was determined with HPLC-DAD detection. Analysis revealed the strongest uptake in the first $2 \mathrm{~h}$ of incubation. After $2 \mathrm{~h}$, the concentration of non-conjugated paracetamol reached its maximum and showed no further increase. In the growth medium, a decrease in the paracetamol concentration of $70 \%$ was observed over the $3 \mathrm{~h}$ of incubation (Fig. 1).

\subsection{Glutathione-dependent detoxification}

LC-MS/MS analysis of extracts of A. rusticana root cells that were dosed with acetaminophen for $3 \mathrm{~h}$ and then transferred to fresh media revealed the formation of two polar metabolites of acetaminophen that were well separated from each other as well as from the acetaminophen itself on the gradient HPLC system. All three analytes showed good peak shapes and signal/noise ratios (Fig. 2). The metabolites detected were acetaminophen-glutathione and acetaminophen-cysteine with their pseudomolecular ions $\left([\mathrm{M}+\mathrm{H}]^{+}\right)$at $\mathrm{m} / \mathrm{z} 457$ and $\mathrm{m} / \mathrm{z} 271$. The identification of these metabolites was based on MS/MS experiments per-

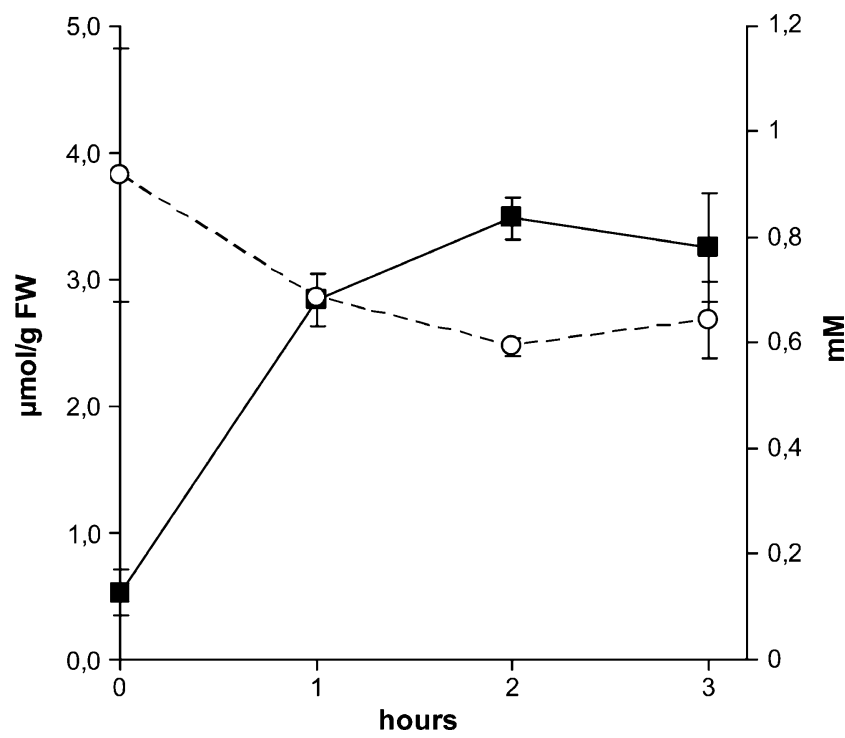

Fig. 1 Amount of free acetaminophen in root cells of A. rusticana ( primary $y$-axis, left) and in the growth medium ( $\circ$ secondary $y$-axis, right) during $3 \mathrm{~h}$ of incubation. Values shown are means $\pm \mathrm{SD}$ from three biological replicates and refer to $1 \mathrm{~g}$ of fresh root cells or the concentration of paracetamol $(\mathrm{mM})$ in the medium, respectively 
Fig. 2 Representative chromatogram of a root extract of $A$. rusticana showing the separation of acetaminophen with its glutathione and cysteine conjugates. The peak heights have been normalized to $100 \%$ to illustrate the relative retention times of each of the components and are not representative for the abundance of the compounds in the extract

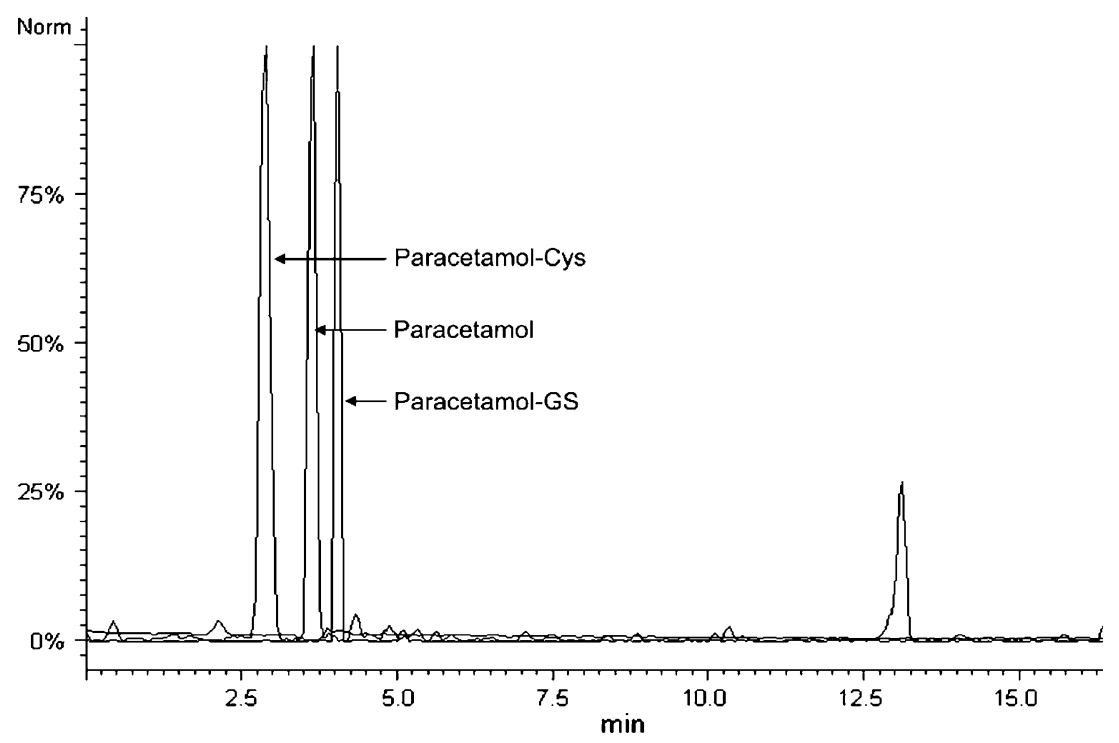

formed in positive ionization mode. Analysis of fragmentation patterns revealed the loss of $129 \mathrm{Da}$, corresponding to the glutamic acid residue of glutathione and resulting in the production of $\mathrm{m} / \mathrm{z}$ 328. For paracetamol-cysteine, the cleavage of the carbon-sulfur bond forms a product ion of $m / z 182$ (Fig. 3). Both spectra show exactly the product ions generated and described by Mutlib et al. (2000). Acetaminophen itself with its pseudomolecular ion $\left([\mathrm{M}+\mathrm{H}]^{+}\right)$of 152 shows a major fragment of $\mathrm{m} / \mathrm{z} 110$, corresponding to the ion $\left(\mathrm{NH}_{3}-\mathrm{C}_{6} \mathrm{H}_{4}-\mathrm{OH}\right)^{+}$obtained after a $\mathrm{CO}=\mathrm{CH}_{2}$ neutral loss.

3.3 Formation of an acetaminophen glucose conjugate

\subsubsection{Mass spectral analysis}

Next to the prominent conjugation reaction with glutathione as a reaction partner, acetaminophen was observed
Fig. 3 MS/MS spectra of $[\mathrm{M}+\mathrm{H}]^{+}$ions of a paracetamolGS $(m / z 457)$ and b paracetamol-Cys $(\mathrm{m} / \mathrm{z} 271)$. The product ion $m / z 328$ is formed by an elimination of glutamic acid, the cleavage of the $\mathrm{C}-\mathrm{S}$ bond results in the formation of $\mathrm{m} / \mathrm{z}$ 182. The mass spectral analysis was done in the positive ionization mode
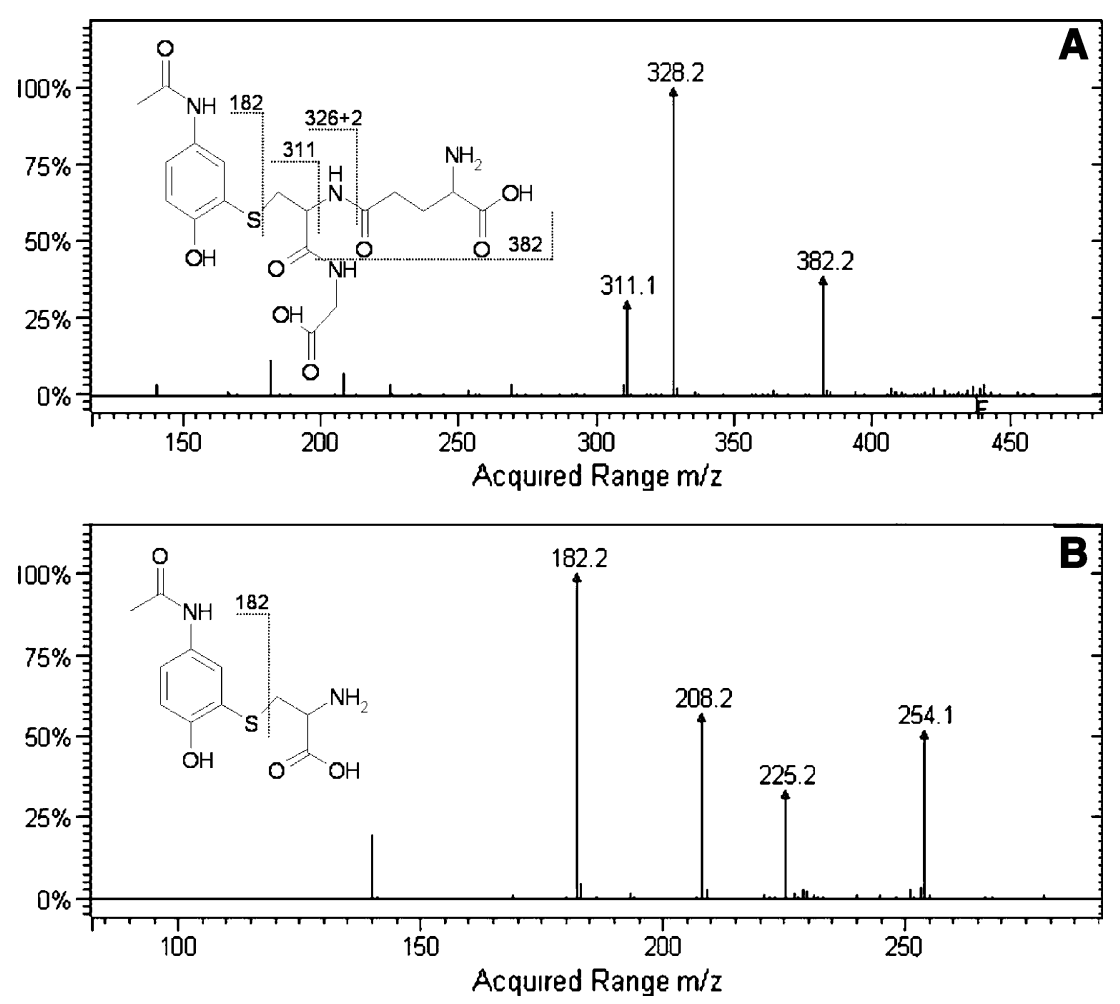
to form a glucoside in root cells of $A$. rusticana. Due to its polarity, the acetaminophen-glucoside elutes earlier than all other acetaminophen-related compounds (RT 1.7 min; Fig. 4a, b, upper chromatograms). Acetaminophen and $1 \mathrm{U}$ of glucose form a glucoside with a pseudomolecular ion $\left([\mathrm{M}+\mathrm{H}]^{+}\right)$of $\mathrm{m} / z$ 314. O-glucosides usually show the loss of $162 \mathrm{Da}$ due to the loss of dehydroglucose $\left(\mathrm{C}_{6} \mathrm{H}_{10} \mathrm{O}_{5}\right)$ during MS/MS fragmentations (Rauha et al. 2001), resulting in the pseudomolecular ion of the parent compound. In case of the acetaminophenglucoside, the first fragmentation reveals the signal of $[\mathrm{M}$ $+\mathrm{H}]^{+} m / z 152$ representing the acetaminophen itself. To further confirm the chemical structure of an acetaminophen-glucoside, $\mathrm{MS}^{3}$ experiments were performed yielding in the characteristic MS/MS spectra for paracetamol with the main product ion of $\mathrm{m} / z 110(314 \rightarrow 152 \rightarrow 110$; data not shown).

\subsubsection{Enzymatic hydrolysis}

To support the formation of an acetaminophen-glucoside, an enzymatic approach was chosen. The in vivo formed glucoside of acetaminophen is a suitable substrate for $\beta$ glucosidase. Incubation of root extracts of $A$. rusticana (see Section 2) with $100 \mathrm{U}$ of $\beta$-glucosidase resulted in a quantitative hydrolysis of the acetaminophen-glucoside.
Acetaminophen as well as its glucose conjugate were detected in samples of roots harvested $6 \mathrm{~h}$ after the incubation with the paracetamol (see Fig. 4a, upper chromatogram), but after $1 \mathrm{~h}$ of incubation with glucosidase, the first peak, eluting after $1.7 \mathrm{~min}$ and representing the paracetamol-glucose, completely disappears as a result of hydrolyzing the ester bond between glucose and acetaminophen (see Fig. 4a, b, lower chromatograms). Fig. $4 \mathrm{~b}$ represents the chromatograms taken in the SIM mode for $(\mathrm{M}+\mathrm{H})^{+} \mathrm{m} / z 314$ showing only the peak for the acetaminophen-glucoside.

\subsection{Time-dependent formation of acetaminophen conjugates}

To address the question of the initial conjugation reactions, samples were taken right after the incubation and after 2, 4, and $6 \mathrm{~h}$. Due to the fact there are no standards for the glucose and glutathione conjugate available, all results correspond to the ratio of analyte peak areas to internal standard peak area. The amount of free paracetamol is rapidly reduced during the first $2 \mathrm{~h}$ after transferring root cells into acetaminophen-free growth medium. Conjugation occurs right after acetaminophen treatment. The acetaminophen-glucoside as well as the glutathione conjugate were detected in cytosolic extracts right after transferring the roots to the acetaminophen-free growth medium, with
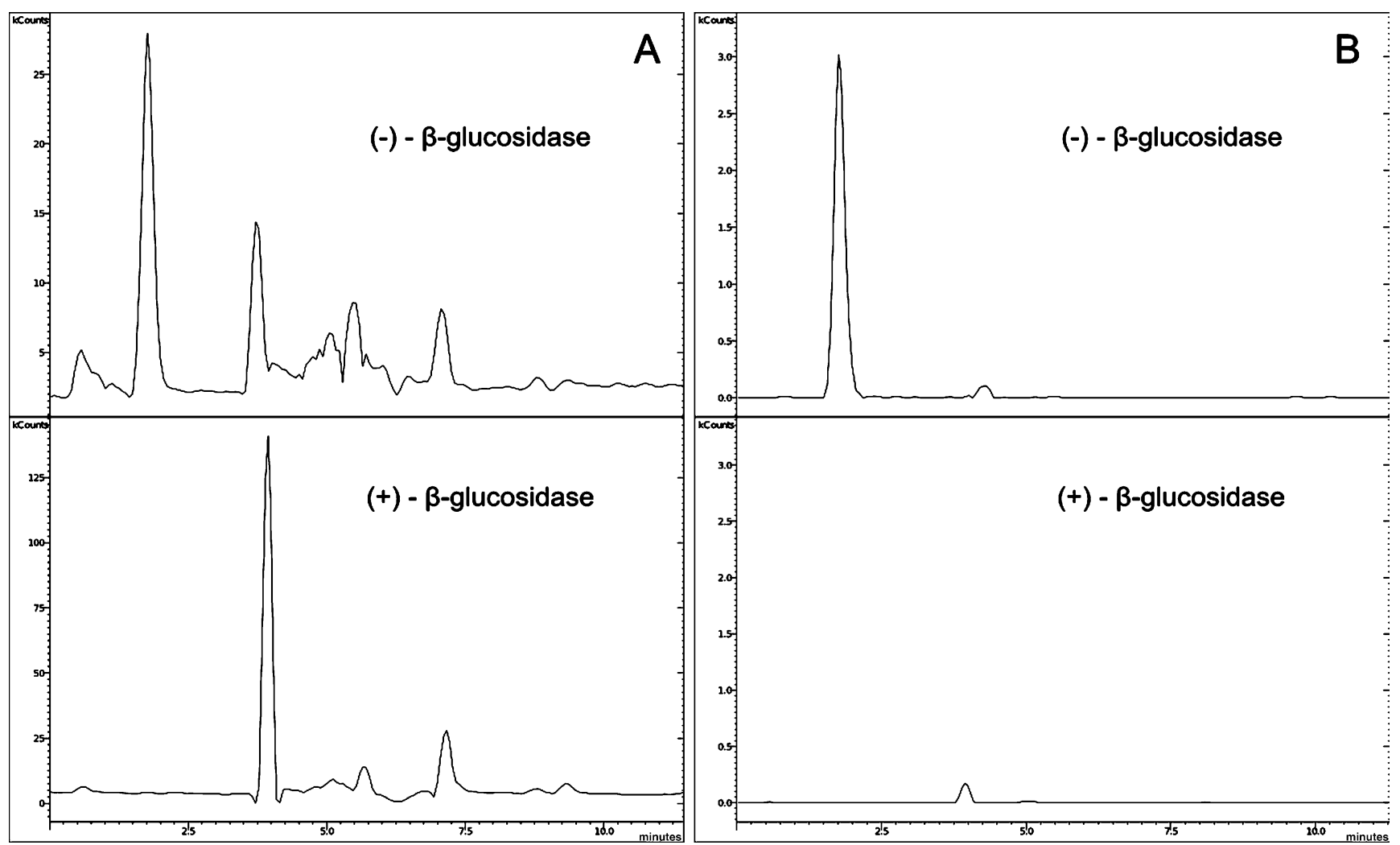

Fig. 4 Chromatograms of samples containing the paracetamol-glucoside. Upper chromatograms represent untreated samples; lower chromatograms show samples that were incubated with $\beta$-D-glucosidase. Scan mode a (TIC), b (SIM for $m / z$ 314) 
acetaminophen-glucoside representing the most dominant metabolite. The concentrations of both conjugates increase over the following $6 \mathrm{~h}$ (Fig. 5). The analysis revealed no acetaminophen-related conjugates in the growth medium (data not shown).

\section{Discussion}

This is one of the first studies addressing the fate of a widely used pharmaceutical in plant tissues. Interestingly, strong similarities to the mammalian system have been observed, which proves the correctness of the green liver concept (Sandermann 1994). Two of the most significant detoxification mechanisms in plants, conjugation with glutathione as well as formation of glucosides, are simultaneously involved in the detoxification of acetaminophen.

This observation points to the direction of two completely independent pathways being involved in plants' acetaminophen metabolism. In mammals, the most abundant acetaminophen metabolite is the glucuronide conjugate representing $40-67 \%$ of the formed conjugates. The rest is either conjugated with sulfate $(20-46 \%)$ or cysteine $(3 \%)$ (Bertolini et al. 2006). A fraction of $5-15 \%$ is further oxidized by enzymes of the P450 mixed-function oxidase system to $N$-acetyl- $p$-benzoquinoneimine [NAPQI] (Bowles et al. 2005; Corcoran et al. 1980) that quickly reacts with glutathione. The resulting paracetamol-glutathione complex is converted to the non-toxic cysteine or mercapturate conjugates, which are eliminated in the urine (Miller et al. 1976).

In plants, a comparable dual detoxification system is involved in the conjugation of paracetamol.

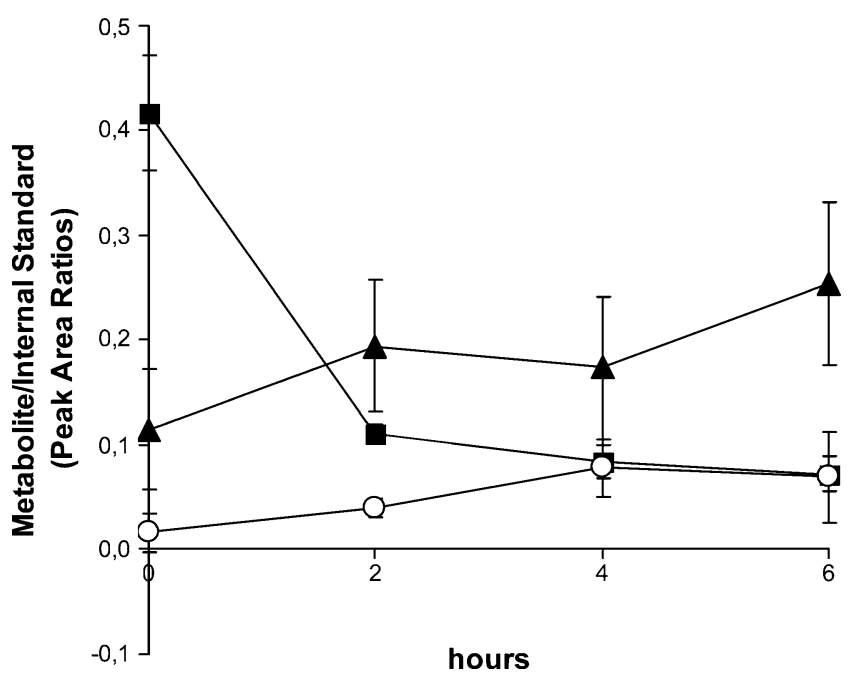

Fig. 5 Relative amounts of acetaminophen (-), acetaminophenglutathione $(\bigcirc)$, and acetaminophen-glycoside $(\boldsymbol{\Delta})$ in root cells after incubation with $1 \mathrm{mM}$ acetaminophen for $3 \mathrm{~h}$. Values shown are means \pm SD from three biological replicates
Glucuronization does not occur in plants, but the corresponding formation of glucosides has been described for a couple of xenobiotics in mammalians (Kirkman et al. 1998). In the described model system of A. rusticana, the paracetamol-glucoside is the dominant metabolite. Based on the molecular structure of the acetaminophen and the characteristic loss of the dehydrosugar, we propose the formation of an O-glucoside, binding the glucose to the hydroxyl group of the benzene ring. The conjugation of paracetamol with $1 \mathrm{U}$ of glucose was already shown for filamentous fungi, separated from soil but not in plants (Huang et al. 2006). The conjugation of free paracetamol to either the glutathione or the glucose conjugate is a rapid process: all conjugation products are present right after $3 \mathrm{~h}$ of incubation. Conjugation with glutathione occurs exclusively on the NAPQI and requires an activation of paracetamol by the P450 monooxygenase enzyme complex. P450 enzymes are known to be present in plants where they catalyze a variety of chemical reactions. Comparable to the mammalian system, the glutathione conjugate represents only a small portion of metabolites. A certain amount of acetaminophen is not conjugated with glucose and is further metabolized to the highly reactive NAPQI intermediate, resulting in the formation of the glutathione conjugate. This reaction is catalyzed by glutathione $S$-transferases.

This formation of a paracetamol-glucoside might be of special interest regarding the use of plants in waste water treatment processes, as glucosides are known precursors to insoluble, bound residues, leaving the xenobiotic compound in a stable and indigestible form bound to the lignin fraction of the cell wall.

We were not able to detect the sulfate conjugate at any time of the experiment in the cells. Sulfotransferases are well-studied plant enzymes that are widely distributed and involved in a broad spectrum of physiological functions (Weinshilboum and Otterness 1994), but with only minor functions in plants' detoxification systems.

\section{Conclusions and perspectives}

With its fast-growing cells in a sterile system, the use of a root cell culture is an appropriate alternative for xenobiotic metabolism studies to evaluate and understand basic detoxification mechanism and set up an analytical method for new xenobiotic compounds in a plant matrix. Hence, our work confirms previous results by Nepovím et al. (2004) who used this cell culture as a model to explore plant metabolism of explosives in the past.

Most of the analytic work in this study was performed on an LC-MS system that represents a powerful tool in identifying and characterizing xenobiotic metabolites (Oliveira and Watson 2000). With no analytic standards 
available, an identification of unknown metabolites with HPLC-UV/VIS technique is not possible. Identification of unknown peaks, formed after incubation with acetaminophen, was hence based on $\mathrm{MS}^{\mathrm{n}}$ experiments.

With the advantages mentioned above, the results indicate that the use of phytoremediation techniques will be of high benefit on waste water treatment facilities and that selected plant species will be able to improve the effluent quality significantly. The resulting plant biomass will contain the detoxified metabolites in soluble and insoluble form and should be stored dry, or incinerated or fermented in a closed compartment to yield bioenergy (Schröder et al. 2008).

With the advantages mentioned above, the presented results mark the beginning of understanding the fate of acetaminophen in plants. For the future, additional studies with plant species that are suitable for phytoremediation of contaminated waste waters need to be performed, covering the uptake and metabolism as well as the impact of paracetamol and similar compounds on the plant stress defense system.

Acknowledgements B. B. received funding from the Deutsche Bundesstiftung Umwelt (DBU). Authors thank Thomas Vanek (Institute of Organic Chemistry and Biochemistry, Academy of Sciences of the Czech Republic) for the A. rusticana cell culture. This work was stimulated by the EU COST Action 859: Phytotechnologies to promote sustainable land use and improve food safety.

Open Access This article is distributed under the terms of the Creative Commons Attribution Noncommercial License which permits any noncommercial use, distribution, and reproduction in any medium, provided the original author(s) and source are credited.

\section{References}

Bertolini A, Ferrari A, Ottani A, Guerzoni S, Tacchi R et al (2006) Paracetamol: new vistas of an old drug. CNS Drug Reviews 12:250-275

Bowles D, Isayenkova J, Lim EK, Poppenberger B (2005) Glycosyltransferases: managers of small molecules. Curr Opin Plant Biol $8: 254-263$

Corcoran GB, Mitchell JR, Vaishnav YN, Horning EC (1980) Evidence that acetaminophen and N-hydroxyacetaminophen form a common arylating intermediate, N-acetyl-p-benzoquinoneimine. Mol Pharmacol 18:536-542

Crane M, Watts C, Boucard T (2006) Chronic aquatic environmental risks from exposure to human pharmaceuticals. Sci Total Environ 367:23-41

Edwards R, Dixon DP (2004) Metabolism of natural and xenobiotic substrates by the plant glutathione S-transferase superfamily. Ecol Stud 170:17-50
Henschel KP, Wenzel A, Diedrich M, Fliedner A (1997) Environmental hazard assessment of pharmaceuticals. Regul Toxicol Pharmacol 25(3):220-225

Hou BK, Lim EK, Higgins GS, Bowles DJ (2004) N-glucosylation of cytokinins by glycosyltransferases of Arabidopsis thaliana. J Biol Chem 279:47822-47832

Huang HH, Lin LH, Zhang P, Qi XL, Zhong DF (2006) Formation of glucoside conjugate of acetaminophen by fungi separated from soil. Eur J Drug Metab Pharmacokinet 31:103-108

Kirkman SK, Zhang MY, Horwatt PM, Scatina J (1998) Isolation and identification of bromfenac glucoside from rat bile. Drug Metab Dispos 26:720-723

Kolpin DW, Furlong ET, Meyer MT, Thurman EM, Zaugg SD et al (2002) Response to comment on, 'Pharmaceuticals, hormones, and other organic wastewater contaminants in US streams, 1999-2000: a national reconnaissance'. Environ Sci Technol 36:4007-4008

Messner B, Thulke O, Schaffner AR (2003) Arabidopsis glucosyltransferases with activities toward both endogenous and xenobiotic substrates. Planta 217:138-146

Miller RP, Roberts RJ, Fischer LJ (1976) Acetaminophen elimination kinetics in neonates, children, and adults. Clin Pharmacol Ther 19:284-294

Mutlib AE, Shockcor J, Espina R, Graciani N, Du A et al (2000) Disposition of glutathione conjugates in rats by a novel glutamic acid pathway: characterization of unique peptide conjugates by liquid chromatography/mass spectrometry and liquid chromatography/NMR. J Pharmacol Exp Ther 294:735-745

Nepovím A, Podlipná R, Soudek P, Schröder P, Vanek T (2004) Effects of heavy metals and nitroaromatic compounds on horseradish glutathione S-transferase and peroxidase. Chemosphere 57:1007-1015

Oliveira EJ, Watson DG (2000) Liquid chromatography-mass spectrometry in the study of the metabolism of drugs and other xenobiotics. Biomed Chromatogr 14:351-372

Rauha JP, Vuorela H, Kostiainen R (2001) Effect of eluent on the ionization efficiency of flavonoids by ion spray, atmospheric pressure chemical ionization, and atmospheric pressure photoionization mass spectrometry. J Mass Spectrom 36:1269-1280

Richman A, Swanson A, Humphrey T, Chapman R, McGarvey B et al (2005) Functional genomics uncovers three glucosyltransferases involved in the synthesis of the major sweet glucosides of Stevia rebaudiana. Plant J 41:56-67

Salomon M (2005) Arzneimittel in der aquatischen Umwelt. Umweltwiss Schadst Forsch 17(1):50-53

Sandermann H (1994) Higher plant metabolism of xenobiotics: the 'green liver' concept. Pharmacogenetics 4:225-241

Schröder P, Khalvati M, Neustifter J, Gschlössl T (2008) Use of Phragmites australis for phytoremediation of organic compounds in municipal waste water treatment plants. Phytoremediation:. In: Navarro-Aviñó JP (ed) The green salvation of the world. Research Signpost, Triviandrum, pp 231-242

Schröder P, Navarro-Aviñó J, Azaizeh H, Goldhirsh A, DiGregorio S et al (2007) Using phytoremediation technologies to upgrade waste water treatment in Europe. Environ Sci Pollut Res 14 (7):490-497

Schulte-Oehlmann U, Oehlmann J, Püttmann W (2007) Humanpharmakawirkstoffe in der Umwelt: Einträge, Vorkommen und der Versuch einer Bestandsaufnahme. Umweltwiss Schadst Forsch 19(3):68-179

Weinshilboum R, Otterness D (1994) Sulfotransferase enzymes. Handb Exp Pharmacol 112:45-78 